\title{
PREVISÃO DA CAPACIDADE DE UM SISTEMA HIDRÁULICO FRENTE A DIVERSAS LINHAS DE RECALQUE
}

\author{
R. R. ROCHA, DAMASCENO, J. J. R. e VIEIRA, L. G. M. \\ Universidade Federal de Uberlândia, Faculdade de Engenharia Química \\ E-mail para contato: rosi.rocha28@gmail.com
}

\begin{abstract}
RESUMO - À medida que linhas de recalque são acopladas, a previsão da vazão de sucção de um sistema hidráulico passa a não ser tão trivial, pois a complexidade físicomatemática e a demanda computacional aumentam consideravelmente. Assim, o presente trabalho teve como objetivo desenvolver uma metodologia computacional capaz de prever a capacidade volumétrica de sucção de um sistema hidráulico composto por mais de uma linha de recalque. Para tanto, vazões volumétricas de captação foram previstas frente à quantidade de linhas de recalque acopladas e os respectivos fatores relevantes (comprimento, diâmetro, rugosidade, quota e potência da bomba), relacionados entre si segundo as diretrizes de um Planejamento Composto Central (PCC). Foi possível observar que a vazão de sucção de um sistema hidráulico elevou-se à medida que se aumentou a quantidade das linhas de recalque, mas até a pressão no distribuidor tender às pressões de descarga (interrupção do escoamento).
\end{abstract}

\section{INTRODUÇÃO}

A Equação de Bernoulli é de grande aplicabilidade na Mecânica dos Fluidos, pois permite a previsão da capacidade volumétrica de um sistema hidráulico ou a potência necessária para prover o bombeamento de determinado fluido (Sisson e Pitts, 1979). Na sua concepção original, a Equação de Bernoulli é uma equação algébrica (Equação 1) e consiste numa simplificação da Equação do Movimento para condições físicas especiais, tais como estado estacionário, fluido ideal (efeitos viscosos desprezíveis) e incompressível, ausência de troca de calor e trabalho de eixo com as vizinhanças e escoamento macroscópico em uma direção preferencial (Bird et al. 2004).

$$
\frac{P_{1}}{\rho g}+\frac{v_{1}^{2}}{2 g}+z_{1}=\frac{P_{2}}{\rho g}+\frac{v_{2}^{2}}{2 g}+z_{2}
$$

Nas condições ideais da Equação 1, bastaria que o usuário conhecesse apenas as condições operacionais $(\mathrm{P}, \mathrm{v}, \mathrm{z})$ no início (1) e término (2) do escoamento para se ter uma descrição física generalizada do sistema. Todavia, nos casos reais, a Equação 1 deve ser reescrita contabilizando as perdas de carga $\left(\mathrm{h}_{\mathrm{C}}\right)$, por causa das dissipações viscosas em tubulações e acessórios, e também pela ação de bombas $\left(\mathrm{h}_{\mathrm{B}}\right)$ e turbinas $\left(\mathrm{h}_{\mathrm{T}}\right)$ eventualmente inseridas no sistema hidráulico (Bennet e Myers, 1978). Nestas hipóteses, a Equação de Bernoulli deve ser reescrita nos termos da Equação (2).

$$
\frac{\mathrm{P}_{1}}{\rho \mathrm{g}}+\frac{\mathrm{v}_{1}^{2}}{2 \mathrm{~g}}+\mathrm{z}_{1}+\mathrm{h}_{\mathrm{B}}=\frac{\mathrm{P}_{2}}{\rho \mathrm{g}}+\frac{\mathrm{v}_{2}^{2}}{2 \mathrm{~g}}+\mathrm{z}_{2}+\mathrm{h}_{\mathrm{C}}+\mathrm{h}_{\mathrm{T}}
$$


Na prática, as cargas de bombas ou turbinas são informações bem-conhecidas e advindas dos respectivos fabricantes, sem maiores dificuldades para seu levantamento. Por sua vez, a avaliação da perda de carga - distribuída ou localizada - depende de equações ou coeficientes empíricos apropriados que são geralmente disponibilizados pela literatura. Quase sempre, a Equação de Bernoulli (Equação 2) é aplicada em situações nas quais a massa de fluido se conserva ao longo do escoamento, haja vista haver apenas uma entrada e uma única saída no sistema analisado. Evidentemente que, nestas condições, a manipulação algébrica da Equação de Bernoulli torna-se perfeitamente factível, sem maiores problemas nas previsões de qualquer um dos seus termos.

Diferentemente dos casos em que há apenas uma entrada e uma única saída de fluido no sistema, a multiplicidade de sucções ou de recalques podem complicar a descrição do fenômeno sob a óptica da Equação de Bernoulli. Apesar de globalmente a massa de fluido se conservar, a multiplicidade de sucções ou recalques promovem a adição ou divisão das vazões mássicas em pontos específicos do sistema. Logo, em função destas adições ou divisões da vazão, certamente haverá também um aumento considerável no número de Equações a ser resolvido.

Isto posto, este trabalho teve como objetivo principal verificar a influência do efeito da multiplicidade de linhas de recalque sob a capacidade de sucção de um determinado sistema hidráulico. Para tanto, o número de linhas de recalque foi combinado com outros fatores considerados relevantes (comprimento, diâmetro, quota, rugosidade, potência de bombeamento) sob a forma de um Planejamento Composto Central (PCC), a fim de que fosse possível a proposição, inclusive, de uma equação de projeto para prever a capacidade volumétrica sob determinadas condições operacionais.

\section{METODOLOGIA}

Na Figura 1 está disposta uma representação esquemática e genérica de um sistema hidráulico em que há uma sucção, uma bomba, um distribuidor e $\mathrm{N}$ linhas de recalque.

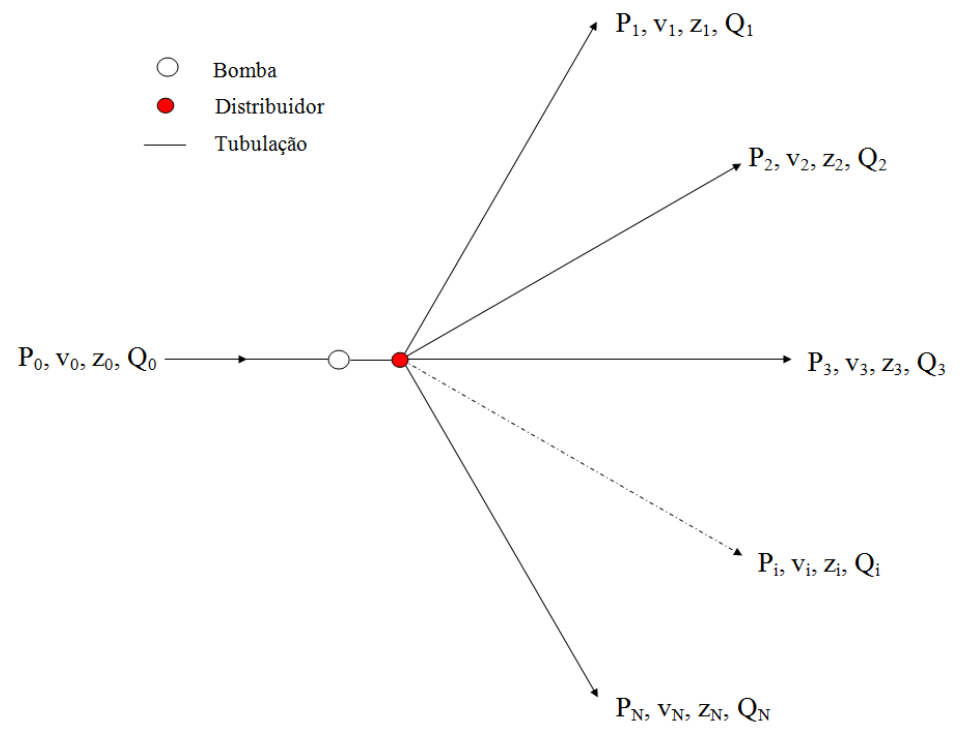

Figura 1 - Representação esquemática de um sistema hidráulico com múltiplas linhas de recalque. 
Nos cálculos efetuados neste trabalho, uma hipótese adotada foi considerar que a pressão do fluido no interior do distribuidor era uniformemente distribuída $\left(\mathrm{P}_{\mathrm{D}}\right)$. Assim, uma equação de Bernoulli foi gerada da sucção até a entrada do distribuidor (primeira linha da matriz da Equação 4) e mais $\mathrm{N}$ equações de Bernoulli foram geradas de cada uma das saídas do distribuidor até as descargas dos respectivos recalques, totalizando, assim, $(\mathrm{N}+1)$ equações. Então, para cada uma das $(\mathrm{N}+1)$ equações de Bernoulli (Equação 4$)$, outras $(\mathrm{N}+1)$ equações também foram geradas para previsão dos respectivos coeficientes de atrito de acordo com a Equação de Colebrook-White $(\mathrm{CW})$ - Equação 5, totalizando, portanto, 2(N+1) equações matemáticas.

$$
\begin{aligned}
& \text { Bernoulli }=\left(\begin{array}{c}
\frac{P_{0}}{\rho g}+z_{0}+h_{B}-\frac{P_{D}}{\rho g}-z_{D}-\left(f_{0} \frac{L_{0}}{D_{0}}+K_{S-0}\right) \frac{v_{0}^{2}}{2 g}=0 \\
\frac{P_{D}}{\rho g}+z_{D}-\frac{P_{1}}{\rho g}-z_{1}-\left(f_{1} \frac{L_{1}}{D_{1}}+K_{S-1}\right) \frac{v_{1}^{2}}{2 g}=0 \\
\frac{P_{D}}{\rho g}+z_{D}-\frac{P_{2}}{\rho g}-z_{2}-\left(f_{2} \frac{L_{2}}{D_{2}}+K_{S-2}\right) \frac{v_{2}^{2}}{2 g}=0 \\
\vdots \\
\frac{P_{D}}{\rho g}+z_{D}-\frac{P_{i}}{\rho g}-z_{i}-\left(f_{i} \frac{L_{i}}{D_{i}}+K_{S-i}\right) \frac{v_{i}^{2}}{2 g}=0 \\
\vdots \\
\frac{P_{D}}{\rho g}+z_{D}-\frac{P_{N}}{\rho g}-z_{N}-\left(f_{N} \frac{L_{N}}{D_{N}}+K_{S-N}\right) \frac{v_{N}^{2}}{2 g}=0
\end{array}\right) \\
& \mathrm{CW}=\left(\begin{array}{c}
\frac{1}{\sqrt{\mathrm{f}_{0}}}+2 \log \left(\frac{\varepsilon_{0} / \mathrm{D}_{0}}{3,7}+\frac{2,5}{\mathrm{Re}_{0} \sqrt{\mathrm{f}_{0}}}\right)=0 \\
\frac{1}{\sqrt{\mathrm{f}_{1}}}+2 \log \left(\frac{\varepsilon_{1} / \mathrm{D}_{1}}{3,7}+\frac{2,5}{\mathrm{Re}_{1} \sqrt{\mathrm{f}_{1}}}\right)=0 \\
\frac{1}{\sqrt{\mathrm{f}_{2}}}+2 \log \left(\frac{\varepsilon_{2} / \mathrm{D}_{2}}{3,7}+\frac{2,5}{\mathrm{Re}_{2} \sqrt{\mathrm{f}_{2}}}\right)=0 \\
\frac{1}{\sqrt{\mathrm{f}_{\mathrm{i}}}}+2 \log \left(\frac{\varepsilon_{\mathrm{i}} / \mathrm{D}_{\mathrm{i}}}{3,7}+\frac{2,5}{\operatorname{Re}_{\mathrm{i}} \sqrt{\mathrm{f}_{\mathrm{i}}}}\right)=0 \\
\vdots \\
\frac{1}{\sqrt{\mathrm{f}_{\mathrm{N}}}}+2 \log \left(\frac{\varepsilon_{\mathrm{N}} / \mathrm{D}_{\mathrm{N}}}{3,7}+\frac{2,5}{\operatorname{Re}_{\mathrm{N}} \sqrt{\mathrm{f}_{\mathrm{N}}}}\right)=0
\end{array}\right)
\end{aligned}
$$

No rol de equações anteriores (Equações 4 e 5), considerando que todas as condições operacionais sejam bem-conhecidas (fluido, diâmetros, comprimentos, rugosidades, quotas, 
potência de bombeamento, pressão de sucção e pressões de descargas, coeficientes de perda), pôde-se afirmar que restaram $(\mathrm{N}+1)$ velocidades médias desconhecidas, $(\mathrm{N}+1)$ fatores de atrito desconhecidos e (1) pressão no distribuidor desconhecida, totalizando, assim, [2(N+1)+1] incógnitas. Cabe ressaltar que na hipótese anterior, o número de incógnitas foi maior que o de equações disponíveis, o que inviabilizaria a obtenção de uma solução única para o problema. Desta forma, neste tipo de problema, a equação que possibilitou a igualdade entre a quantidade de equações e de incógnitas foi dada por uma restrição de natureza física, oriunda do balanço global de material para um fluido incompressível.

$$
\mathrm{Q}_{0}=\sum_{\mathrm{i}=1}^{\mathrm{N}} \mathrm{Q}_{\mathrm{i}}
$$

Para tanto, o conjunto de equações não-lineares apresentado anteriormente foi resolvido no software Maple ${ }^{\circledR}$ mediante a aplicação do Método Floating-Point Arithmetic. A fim de evitar que o rol de equações anteriormente mencionado fosse aleatoriamente resolvido, este trabalho optou por organizar os fatores operacionais relevantes sobre a forma de um Planejamento Composto Central (PCC), nos termos da Matriz de Planejamento (Box et al., 1978) apresentada na Tabela 1 (fatores codificados). Os fatores operacionais relevantes foram a quantidade de linhas de recalque $(2 \leq \mathrm{N}(-) \leq 10)$, o comprimento das linhas de recalque $(100 \leq \mathrm{L}(\mathrm{m}) \leq 1000)$, o diâmetro da tubulação das linhas de recalque $(1 \leq \mathrm{D}$ (in) $\leq 5)$, as quotas das descargas das linhas dos recalques $(-200 \leq \mathrm{z}(\mathrm{m}) \leq 200)$, a potência de bombeamento $\left(5 \leq \mathrm{P}_{\mathrm{B}}(\mathrm{hp}) \leq 15\right)$ e a rugosidade relativa das linhas de recalque $(0,0000 \leq \varepsilon / \mathrm{D}(-) \leq 0,0500)$, as quais receberam a denominação de $\mathrm{X}_{1}, \mathrm{X}_{2}, \mathrm{X}_{3}$, $\mathrm{X}_{4}, \mathrm{X}_{5}$ e $\mathrm{X}_{6}$, respectivamente.

Tabela 1 - Matriz de Planejamento (ortogonalidade $(\alpha)$ igual a 2)

\begin{tabular}{|c|c|c|c|c|c|c|c|c|c|c|c|c|c|}
\hline \multirow[b]{2}{*}{$\mathrm{n}$} & \multicolumn{6}{|c|}{ Fatores } & \multirow[b]{2}{*}{$\mathrm{n}$} & \multicolumn{6}{|c|}{ Fatores } \\
\hline & $\mathrm{X}_{1}$ & $\mathrm{X}_{2}$ & $\mathrm{X}_{3}$ & $\mathrm{X}_{4}$ & $\mathrm{X}_{5}$ & $\mathrm{X}_{6}$ & & $\mathrm{X}_{1}$ & $\mathrm{X}_{2}$ & $X_{3}$ & $\mathrm{X}_{4}$ & $\mathrm{X}_{5}$ & $\mathrm{X}_{6}$ \\
\hline 01 & -1 & -1 & -1 & -1 & -1 & -1 & 24 & +1 & -1 & +1 & +1 & +1 & -1 \\
\hline 02 & -1 & -1 & -1 & -1 & +1 & +1 & 25 & +1 & +1 & -1 & -1 & -1 & -1 \\
\hline 03 & -1 & -1 & -1 & +1 & -1 & +1 & 26 & +1 & +1 & -1 & -1 & +1 & +1 \\
\hline 04 & -1 & -1 & -1 & +1 & +1 & -1 & 27 & +1 & +1 & -1 & +1 & -1 & +1 \\
\hline 05 & -1 & -1 & +1 & -1 & -1 & +1 & 28 & +1 & +1 & -1 & +1 & +1 & -1 \\
\hline 06 & -1 & -1 & +1 & -1 & +1 & -1 & 29 & +1 & +1 & +1 & -1 & -1 & +1 \\
\hline 07 & -1 & -1 & +1 & +1 & -1 & -1 & 30 & +1 & +1 & +1 & -1 & +1 & -1 \\
\hline 08 & -1 & -1 & +1 & +1 & +1 & +1 & 31 & +1 & +1 & +1 & +1 & -1 & -1 \\
\hline 09 & -1 & +1 & -1 & -1 & -1 & +1 & 32 & +1 & +1 & +1 & +1 & +1 & +1 \\
\hline 10 & -1 & +1 & -1 & -1 & +1 & -1 & 33 & $-\alpha$ & 0 & 0 & 0 & 0 & 0 \\
\hline 11 & -1 & +1 & -1 & +1 & -1 & -1 & 34 & $+\alpha$ & 0 & 0 & 0 & 0 & 0 \\
\hline 12 & -1 & +1 & -1 & +1 & +1 & +1 & 35 & 0 & $-\alpha$ & 0 & 0 & 0 & 0 \\
\hline 13 & -1 & +1 & +1 & -1 & -1 & -1 & 36 & 0 & $+\alpha$ & 0 & 0 & 0 & 0 \\
\hline 14 & -1 & +1 & +1 & -1 & +1 & +1 & 37 & 0 & 0 & $-\alpha$ & 0 & 0 & 0 \\
\hline 15 & -1 & +1 & +1 & +1 & -1 & +1 & 38 & 0 & 0 & $+\alpha$ & 0 & 0 & 0 \\
\hline 16 & -1 & +1 & +1 & +1 & +1 & -1 & 39 & 0 & 0 & 0 & $-\alpha$ & 0 & 0 \\
\hline 17 & +1 & -1 & -1 & -1 & -1 & +1 & 40 & 0 & 0 & 0 & $+\alpha$ & 0 & 0 \\
\hline 18 & +1 & -1 & -1 & -1 & +1 & -1 & 41 & 0 & 0 & 0 & 0 & $-\alpha$ & 0 \\
\hline 19 & +1 & -1 & -1 & +1 & -1 & -1 & 42 & 0 & 0 & 0 & 0 & $+\alpha$ & 0 \\
\hline 20 & +1 & -1 & -1 & +1 & +1 & +1 & 43 & 0 & 0 & 0 & 0 & 0 & $-\alpha$ \\
\hline 21 & +1 & -1 & +1 & -1 & -1 & -1 & 44 & 0 & 0 & 0 & 0 & 0 & $+\alpha$ \\
\hline 22 & +1 & -1 & +1 & -1 & +1 & +1 & 45 & 0 & 0 & 0 & 0 & 0 & 0 \\
\hline 23 & +1 & -1 & +1 & +1 & -1 & +1 & $46(5)$ & 0 & 0 & 0 & 0 & 0 & 0 \\
\hline
\end{tabular}


As respectivas equações de codificação dos fatores mencionados na Tabela 2 encontram-se representadas pela Equação 7 .

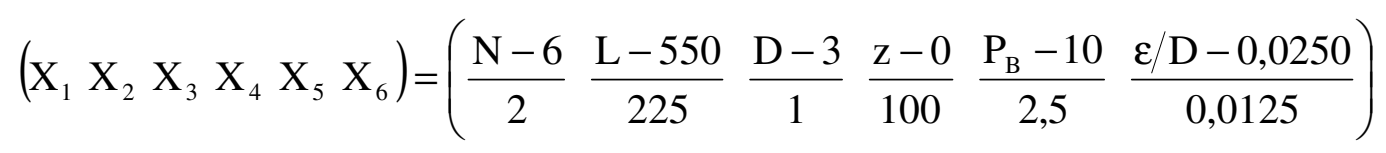

De acordo com as condições operacionais contidas em cada uma das linhas da Matriz de Planejamento (Tabela 1), as Equações 4 a 6 foram resolvidas de modo a fornecer a velocidade média do fluido em cada trecho de tubulação, os fatores de atritos e a pressão no distribuidor. Então, para cada linha da matriz, a partir das velocidades médias do fluido, foi possível estimar as respectivas vazões volumétricas em cada uma das tubulações de recalque (resposta calculada) que somadas resultaram a resposta almejada (vazão volumétrica de sucção). Após esta etapa da estimativa das vazões volumétricas de sucção $\left(\mathrm{Q}_{0}\right)$, Técnicas de Regressão Múltipla foram aplicadas para se obter a Superfície de Resposta (Myers, 1976), genericamente representada pela Equação 8, na qual contempla o valor médio, os efeitos lineares (vetor b), os efeitos quadráticos (diagonal principal da matriz B) e os efeitos de interação (elementos fora da diagonal principal da matriz B).

$$
\mathrm{Q}_{0}=\beta+\mathrm{bX}+\mathrm{X}^{\mathrm{T}} \mathrm{BX}
$$

Por fim, no presente trabalho, água à temperatura de $20^{\circ} \mathrm{C}$ foi utilizada como fluido. Tanto a pressão de sucção $\left(\mathrm{P}_{0}\right)$ quanto as pressões de descargas foram consideradas como se estivessem à pressão atmosférica. Todas as descargas receberam a mesma quota conforme os valores de cada linha (n) da Matriz de Planejamento. Nos demais cálculos, o comprimento da tubulação à montante do distribuidor foi considerada bem menor (desprezível) do que as dimensões lineares das linhas de recalque (à jusante do distribuidor).

\section{RESULTADOS}

$\mathrm{Na}$ Figura 2, são apresentadas as vazões volumétricas de sucção $\left(\mathrm{Q}_{0}\right)$ obtidas nas condições operacionais contidas em cada uma das linhas (n) da Matriz de Experimento (Tabela 1).

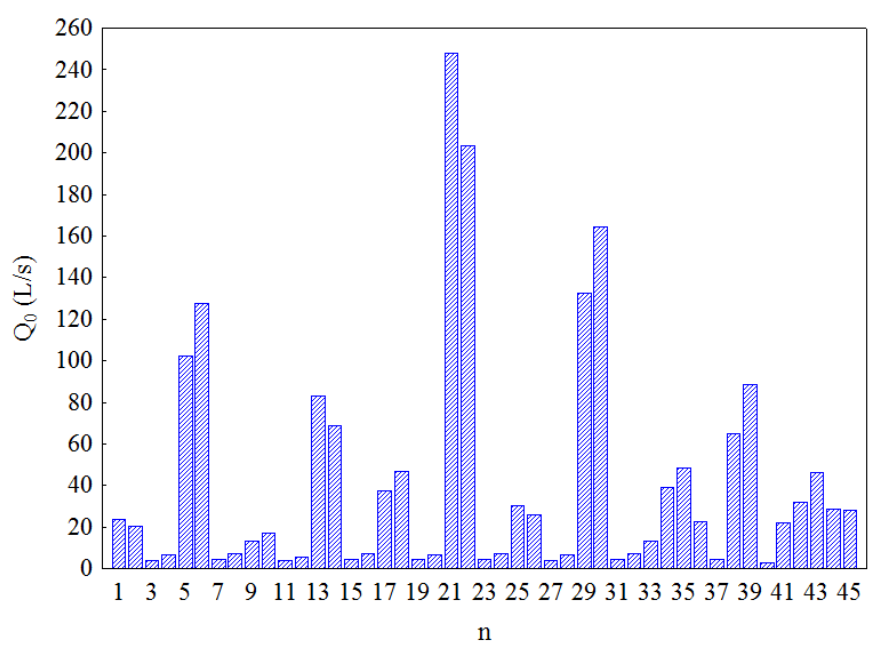

Figura 2 - Vazões volumétricas de sucção para cada uma das n linhas da Matriz de Planejamento. 
De acordo com a Figura 2, pôde-se observar que, a depender das condições operacionais utilizadas, o sistema hidráulico proposto para este trabalho reagiu de maneira distinta e captou diferentes capacidades volumétricas que estiverem entre 2 e $250 \mathrm{~L} / \mathrm{s}$. Em face da quantidade significativa de fatores utilizados (seis), a fim de analisar os efeitos que cada um exerceu sobre este tipo de problema, Técnicas de Regressão Múltipla foram aplicadas sobre tais dados e uma Superfície de Resposta foi obtida para a descrição do fenômeno, conforme mostram as Equações 9,10 e 11. Vale ressalvar que foram considerados apenas os efeitos que tiveram nível de significância menor ou igual a 5\%.

$$
\begin{aligned}
\mathrm{Q}_{0} & =35,88+\mathrm{b}\left(\begin{array}{l}
\mathrm{X}_{1} \\
\mathrm{X}_{2} \\
\mathrm{X}_{3} \\
\mathrm{X}_{4} \\
\mathrm{X}_{5} \\
\mathrm{X}_{6}
\end{array}\right)+\left(\begin{array}{llllll}
\mathrm{X}_{1} & \mathrm{X}_{2} & \mathrm{X}_{3} & \mathrm{X}_{4} & \mathrm{X}_{5} & \mathrm{X}_{6}
\end{array}\right) \mathrm{B}\left(\begin{array}{l}
\mathrm{X}_{1} \\
\mathrm{X}_{2} \\
\mathrm{X}_{3} \\
\mathrm{X}_{4} \\
\mathrm{X}_{5} \\
\mathrm{X}_{6}
\end{array}\right) \\
\mathrm{b} & =\left(\begin{array}{ccccccc}
+12,15 & -8,19 & +25,95 & -35,72 & 0 & 0
\end{array}\right) \\
\mathrm{B} & =\left(\begin{array}{cccccc}
0 & 0 & +4,67 & -6,74 & 0 & 0 \\
0 & 0 & -2,96 & +4,29 & 0 & 0 \\
+4,67 & -2,96 & 0 & -14,2 & 0 & 0 \\
-6,74 & +4,29 & -14,2 & +5,66 & 0 & 0 \\
0 & 0 & 0 & 0 & 0 & 0 \\
0 & 0 & 0 & 0 & 0 & 0
\end{array}\right)
\end{aligned}
$$

De acordo com a Superfície de Resposta, a primeira constatação foi observar que dois fatores tiveram todos seus efeitos (lineares, quadráticos e de interação) irrelevantes (nulos) no que se refere à capacidade de sucção do sistema hidráulico estudado. Tais fatores foram a potência de bombeamento $\left(\mathrm{X}_{5}\right)$ e a rugosidade relativa $\left(\mathrm{X}_{6}\right)$. Dentre os fatores relevantes, constatou-se que o mais importante foi a quota da descarga $\left(\mathrm{X}_{4}\right)$, seguido, em ordem decrescente, pelo diâmetro da tubulação de recalque $\left(\mathrm{X}_{3}\right)$, pelo número de linhas de recalque $\left(\mathrm{X}_{1}\right)$ e pelo comprimento da tubulação de recalque $\left(\mathrm{X}_{2}\right)$. $\mathrm{O}$ número de linhas de recalque $\left(\mathrm{X}_{1}\right)$ e o diâmetro da tubulação de recalque $\left(\mathrm{X}_{3}\right)$ desempenharam um efeito direito sobre a vazão volumétrica de sucção, ao passo que os outros dois restantes $\left(\mathrm{X}_{2}\right.$ e $\left.\mathrm{X}_{4}\right)$ contribuíram inversamente sobre tal resposta. Em se tratando dos efeitos quadráticos (diagonal principal da matriz $\mathrm{B}$ ), apenas as quotas $\left(\mathrm{X}_{4}\right)$ o apresentaram significativo $(+5,66)$. Foi possível observar ainda que houve alguns efeitos importantes de interação, tais como os efeitos interativos da quota e do diâmetro com o número de linhas e comprimento de tubulação $\left(\mathrm{B}_{41}, \mathrm{~B}_{42}, \mathrm{~B}_{31}\right.$ e $\left.\mathrm{B}_{32}\right)$. Vale mencionar que tais efeitos de interação são representados simetricamente fora da diagonal principal da matriz $\mathrm{B}$, cujos valores são divididos pela metade.

Combinando as informações mencionadas anteriormente, fica evidente o motivo pelos quais as condições operacionais das linhas 21, 20 e 30 da Matriz de Planejamento levaram, nesta ordem, às maiores vazões volumétricas de sucção. Em sentido oposto, justificam-se os mesmos motivos pelos quais as condições operacionais das linhas $3,7,11,14,15,19,23,27,31,37$ e 40 tiveram as menores capacidades de sucção. 
A fim de analisar o efeito apenas do número de linhas de recalque, a linha 45 da Tabela 1 foi escolhida para tal finalidade, mantendo-se o comprimento, diâmetro e quotas das linhas de recalque constantes e adicionando-se linhas de recalque após o distribuidor $(1 \leq \mathrm{N} \leq 100)$. Desta forma, as vazões de sucção $\left(\mathrm{Q}_{0}\right)$ e as vazões individuais de cada uma das linhas de recalque $\left(\mathrm{Q}_{\mathrm{i}}\right)$ são apresentadas na Figura 3. Do mesmo modo, a pressão no distribuidor $\left(\mathrm{P}_{\mathrm{D}}\right)$ também foi acompanhada ao longo da multiplicidade das linhas de recalque $(\mathrm{N})$.
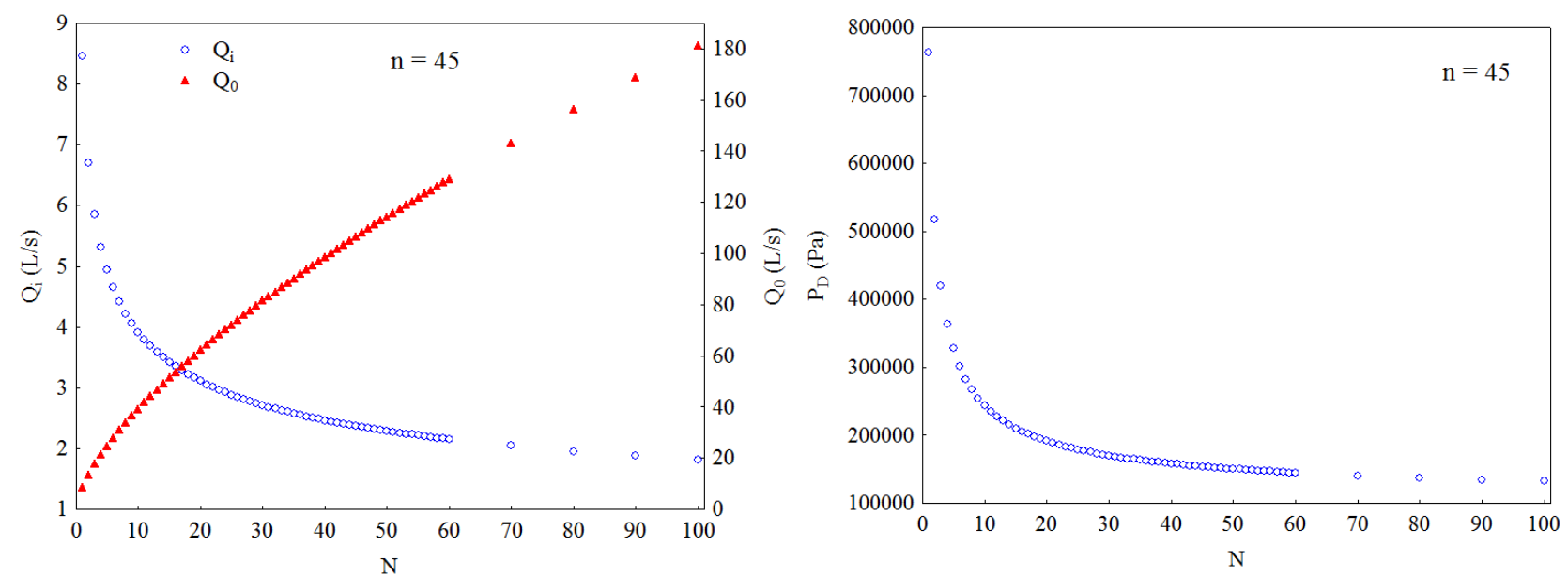

Figura 3 - Efeito da multiplicidade das linhas de recalque $(\mathrm{N})$ sobre a vazão de sucção $\left(\mathrm{Q}_{0}\right)$, as vazões individuais $\left(\mathrm{Q}_{\mathrm{i}}\right)$ e a pressão no distribuidor $\left(\mathrm{P}_{\mathrm{D}}\right)$ para as condições operacionais $\mathrm{n}=45$.

De acordo com a primeira parte da Figura 3, foi possível constatar que a vazão total de sucção foi favorecida à medida em que foram adicionadas linhas de recalque após o distribuidor. Significou dizer que, salvo os custos com a aquisição e instalação de tubulações adicionais, seria possível, com a mesma potência de bombeamento, prover a captação de uma maior vazão de fluido. Por outro lado, apesar do benefício de uma maior captação de fluido, a vazão individual de cada uma das linhas decresceu com a multiplicidade de recalques. Outro aspecto interessante referiu-se à pressão do fluido no distribuidor que decresceu com a multiplicidade de linhas, tendendo a se igualar às pressões de descarga (segunda parte da Figura 3).

Logo, a adição de novas linhas de recalque sofreu basicamente duas limitações: uma de natureza física e duas de natureza operacional. A limitação de natureza física consiste nas dimensões do distribuidor que, por ser finito, impossibilitaria, na prática, a instalação de muitas linhas de recalque. A primeira limitação de natureza operacional consiste no patamar de pressão dentro do distribuidor que, ao tender às pressões de descarga, interromperia o escoamento. A segunda limitação de natureza operacional consiste na demanda requerida em cada uma das linhas de recalque, pois à medida que a multiplicidade ocorre, haveria diminuição da vazão individual que poderia não mais atender às expectativas do usuário. Por fim, a metodologia aqui apresentada foi muito bem-posta, cabendo ao usuário ponderá-la para aproveitar seus benefícios, a fim de que as vantagens trazidas pela multiplicidade de linhas de recalque sobressaiam sobre as desvantagens eventualmente existentes. 


\section{CONCLUSÕES}

De acordo com os principais resultados obtidos neste trabalho, foi possível constatar que a vazão volumétrica de sucção dependeu diretamente do número de linhas de recalque e do diâmetro da tubulação empregado e inversamente do comprimento e quotas das tubulações de recalque. Pôde concluir também que a adição de linhas de recalque acarretou um aumento na vazão volumétrica de sucção do sistema. Todavia, tal benefício deve ser ponderado, pois simultaneamente ocorreu a diminuição da vazão volumétrica de fluido individual para cada uma das linhas de recalque. Por fim, pôde-se obter uma equação de projeto (superfície de resposta) que foi capaz de prever com uma confiabilidade de $93 \%$ a vazão volumétrica de sucção que um sistema hidráulico com múltiplos recalques pode fornecer aos usuários nas condições operacionais empregadas.

\section{NOMENCLATURA}

$\mathrm{D}$ - diâmetro da tubulação das linhas de recalque (in)

$\mathrm{f}$ - fator de atrito (-)

$\mathrm{g}$ - aceleração gravitacional $\left(\mathrm{m} / \mathrm{s}^{2}\right)$

$\mathrm{K}_{\mathrm{S}}$ - coeficiente de perda para cargas localizadas (-)

$\mathrm{L}$ - comprimento da tubulação das linhas de recalque (m)

$\mathrm{n}$ - numeração das linhas da matriz de planejamento (-)

$\mathrm{N}$ - número de linhas de recalque (-)

$\mathrm{P}$ - pressão total do fluido $(\mathrm{Pa})$

$\mathrm{P}_{\mathrm{D}}$ - pressão total do fluido no distribuidor $(\mathrm{Pa})$

$\mathrm{Q}_{\mathrm{i}}$ - vazão volumétrica de fluido em cada uma das linhas de recalque $(\mathrm{L} / \mathrm{s})$

$\mathrm{Q}_{0}$ - vazão volumétrica de fluido na sucção $(\mathrm{L} / \mathrm{s})$

$\mathrm{Re}$ - número de Reynolds

$\mathrm{v}$ - velocidade média de escoamento do fluido $(\mathrm{m} / \mathrm{s})$

$\mathrm{X}$ - codificação de determinado fator

$\mathrm{Z}$ - quota das descargas das linhas de recalque com base na quota do distribuidor (m)

$\beta$ - termo independente da superfície de resposta $(\mathrm{L} / \mathrm{s})$

$\varepsilon$ - rugosidade absoluta da tubulação de recalque $(\mathrm{m})$

$\rho$ - densidade do fluido $\left(\mathrm{kg} / \mathrm{m}^{3}\right)$

\section{AGRADECIMENTOS}

Os autores deste trabalho agradecem à FAPEMIG (PCE-00082/2014) e ao Laboratório de Separação e Energias Renováveis (LASER) da FEQUI/UFU pelos recursos disponibilizados.

\section{REFERÊNCIAS BIBLIOGRÁFICAS}

BENNET, C. O.; MYERS, J. E. Fenômeno de Transporte - Quantidade de Movimento, Calor e Massa, 1978.

BIRD, R. B; STEWART, W. E.; LIGHTFOOT, E. N. Transport Phenomena, 2004.

BOX, M. J.; HUNTER, W. G.; HUNTER, J. S. Statistics for Experiments: an introduction to design, data analysis and model building, John Wiley and Sons, 1978.

MYERS, R. H. Response Surface Methodology, Edwards Brother, 1976.

SISSON, L. E.; PITTS, D. R. Fenômenos de Transporte, Guanabara Dois, 1979. 